\title{
La influencia de los sistemas de vivienda en las condiciones residenciales de la población inmigrante. Los casos de Barcelona y Amsterdam ${ }^{1}$
}

\author{
Albert Terrones Ribas \\ Universitat Autònoma de Barcelona. Departament de Sociologia \\ Grup d'Estudis d'Immigració i Minories Ėtniques (GEDIME) \\ 08193 Bellaterra (Barcelona). Spain
}

\section{Resumen}

Esta investigación se interroga sobre los factores explicativos y los mecanismos productores de las desigualdades y las diferencias en las condiciones residenciales de la población de origen inmigrante. Se hace un énfasis especial a aquéllos relacionados con el sistema de vivienda. Para ello, se desarrolla un estudio comparativo de las condiciones residenciales de los inmigrantes en Barcelona y en Amsterdam. Los resultados muestran como dichos mecanismos y factores son muy diferentes en ambas ciudades. Mientras que en Amsterdam el rol del estado es el factor explicativo clave, en Barcelona este protagonismo recae en el mercado. Muestran también como el impacto sobre la exclusión residencial es mucho más acusado en el caso de Barcelona, como resultado de dichos mecanismos.

Palabras clave: política de vivienda, sistemas de vivienda, minorías étnicas, inmigración, segregación, exclusión residencial, Barcelona, Amsterdam.

\section{Abstract. The influence of the housing systems on residential conditions of immigrants in Barcelona and Amsterdam}

This project aims to detect and explain the factors and mechanisms producing inequality and differences on the residential conditions of immigrants. A special attention is paid to those factors related with the housing systems. In order to do that, the residential conditions of non-western immigrants are compared in two cities: Barcelona and Amsterdam. Results show how there are different mechanisms producing residential differences on both cities. They are mainly based on state regulation in the Amsterdam's case, whereas market seems to be the main factor producing differences Barcelona's case. They show also how residential exclusion has a higher impact in Barcelona as a result of theses mechanisms.

Key words: housing policy, housing system, ethnic minorities, immigration, segregation, residential exclusion, Barcelona, Amsterdam.

1. Esta investigación se enmarca en la Research and Trainning Network UrbEUROPE y fue posible gracias a una estancia de investigación en el Amsterdam Institute for Metropolitan and Development Studies (Universidad de Amsterdam). 
Las condiciones residenciales son un reflejo de la estructura social dominante en cada sociedad. Prueba de ello es la forma como la existencia de un eje de desigualdad por razón de origen étnico se concreta en las condiciones residenciales de la población con origen inmigrante: mayor incidencia de la exclusión residencial, segregación residencial, etc.

Esta investigación se interroga sobre los factores explicativos de las desigualdades y diferencias en las condiciones residenciales de la población con origen inmigrante. El concepto de condiciones residenciales incluye tres dimensiones profundamente interconectadas. Éstas son: a) la dimensión "vivienda», que alude a las características físicas de la vivienda; $b$ ) la dimensión "hogar», que remite a las características del grupo humano que habita la vivienda, junto a la relación de adecuación entre dichas características y las características de la vivienda, y c) la dimensión «localidad», que toma en consideración la ubicación de las viviendas en una determinada estructura urbana. La literatura científica al respecto ha identificado ya un elevado número de factores que intervienen en dicho fenómeno desde diferentes niveles de análisis. Sin embargo, nuestro interés se centra en la evaluación del impacto de las variables relacionadas con el sistema de vivienda, entendido como tal el sistema de relaciones entre los actores que intervienen sobre la producción, el consumo y la regulación de la vivienda. Así pues, atendemos a variables como los mecanismos de provisión de viviendas, las características del mercado y la oferta de vivienda, las disposiciones económicas de los agentes o el papel del estado.

El impacto de dichas variables está establecido a partir del estudio comparativo de las condiciones residenciales de la población con origen inmigrante en dos ciudades: Amsterdam y Barcelona. La metodología incluye la explotación de fuentes estadísticas, técnicas GIS y el estudio bibliográfico de los estudios realizados en ambas ciudades.

Ambas ciudades son representativas de dos sistemas de vivienda claramente diferenciados. De forma general, el sistema holandés — pese a las recientes transformaciones de las que es objeto- guarda paralelismos con lo que Esping Andersen describe como la concepción socialdemócrata del estado de bienestar, mientras que — si atendemos únicamente a la vivienda- el sistema español se encuentra muy próximo a una concepción liberal-corporativista. En los Países Bajos la intervención del Estado en la provisión de vivienda es muy intensa y se encuentra fundamentalmente articulada a través de tres instrumentos: el extenso parque de viviendas de alquiler social (un 54,3\% en 2003); la intervención sobre los precios de la vivienda en alquiler, tanto en el mercado público como en gran parte del mercado privado, y las subvenciones directas al alquiler para los hogares con pocos recursos. Por el contrario, en Barcelona, la familia y, sobre todo, el mercado son las instituciones clave en la provisión de viviendas. El impacto de la débil política de vivienda se ha concretado en la configuración de un mercado ampliamente desregulado, fragmentado y basado en la vivienda en propiedad.

Una primera aproximación a las condiciones residenciales de los hogares de origen inmigrante en ambas ciudades muestra aparentes elementos en 
común, tales como un nivel de segregación residencial moderado, la persistencia de desigualdad en las condiciones residenciales, las diferencias entre diversos grupos de inmigrantes o la segmentación étnica del mercado de vivienda. Dicha segmentación se produce cuando únicamente un determinado segmento del mercado (definido a partir de la localización de la vivienda o las características de la misma) es accesible para la población inmigrada.

Pese a estos aparentes paralelismos, la investigación arroja dos conclusiones. La primera afirma que los mecanismos sociales que determinan las condiciones residenciales de la población inmigrada son muy diferentes en ambas ciudades. Concretamente, en el caso de Amsterdam, el factor explicativo clave es la intervención y la regulación del Estado. Por el contrario, en Barcelona, la explicación debe basarse en las características y la evolución del mercado de la vivienda. La segunda conclusión apunta a que ambos sistemas de provisión residencial provocan efectos muy claros, tanto sobre el nivel como sobre la forma de la exclusión residencial, que afecta a dichos colectivos.

De forma muy breve, durante la década de 1950 y parte de 1960, en Amsterdam, los guest workers (mayoritariamente sureuropeos, norteafricanos y turcos) e inmigrantes procedentes de las antiguas colonias (en especial, Surinam y Antillas), mantenían unas condiciones residenciales parecidas a las de la población inmigrante residente hoy en Barcelona. Las barreras institucionales de acceso al parque de vivienda pública mantenían a dichos trabajadores en pensiones, infraviviendas ofrecidas por los empresarios o en el segmento residual del mercado de alquiler privado. Dichas viviendas se hallaban básicamente dentro del degradado centro urbano o cerca de las áreas industriales. Con el reconocimiento del carácter permanente de la inmigración, se reconoció el derecho de estos inmigrantes a acceder a la amplia oferta de vivienda pública. Esto ocurría, precisamente, en un momento en que, debido al proceso de suburbanización de la población holandesa y la intensidad constructiva, existía una importante oferta vacante de estas viviendas, especialmente en las áreas menos atractivas, como, por ejemplo, Bijlmermeer. Este proceso supuso una mejora importante de las condiciones residenciales de todo el colectivo. Todavía hoy, un $76 \%$ de los surinameses y un $80 \%$ de los turcos y marroquíes se alojan en viviendas de alquiler social ${ }^{2}$. Dicha tipología de viviendas, al contrario que en otras ciudades europeas, no es una oferta aislada geográficamente, residual o estigmatizada. La vivienda social se encuentra distribuida por toda la ciudad, presenta unos niveles de calidad aceptables y una importante heterogeneidad en términos de características o precios. De hecho, aloja a personas con niveles socioeconómicos muy diferentes.

El alto porcentaje de personas de origen inmigrante que habita en viviendas sociales provoca que la distribución de este colectivo en la ciudad dependa de la propia distribución de la vivienda social (muy repartida en el territorio). Sin embargo, los inmigrantes no se encuentran homogéneamente 
distribuidos dentro de la oferta de vivienda social y, por lo tanto, tampoco dentro de la estructura urbana. El resultado es la segregación de dichas minorías.

En el sistema de vivienda holandés, dicho fenómeno no guarda correspondencia con el nivel de renta. De hecho, gracias a la amplia oferta de vivienda pública y a las subvenciones individuales al alquiler ${ }^{3}$, el acceso a la vivienda en Amsterdam mantiene un nivel de desmercantilización muy alto, lo que permite, por ejemplo, la concentración de algunas minorías en los segmentos más caros de la oferta de vivienda pública. Dichas diferencias son fruto de factores como los efectos del sistema de adjudicación de viviendas (que premia a aquellos con mayor capacidad para esperar), las preferencias residenciales o las necesidades de los hogares. Esto último es porque el sistema facilita el acceso a una vivienda más amplia en función del tamaño del hogar. Por ello, turcos y marroquíes, con unos hogares significativamente más extensos, ocupan también la oferta de pisos más amplios y caros, aunque paradójicamente el nivel de recursos netos que deben dedicar a la vivienda es inferior al de otros grupos ${ }^{4}$.

Dicho escenario contrasta con la producción de diferencias residenciales en un contexto más liberal como Barcelona. El predominio absoluto de los mecanismos de mercado en la provisión de vivienda para los inmigrantes, permite un impacto directo sobre las condiciones residenciales de factores como las diferencias en los recursos, la discriminación de los propietarios o los cambios en el mercado de la vivienda. De hecho, las condiciones residenciales de los inmigrantes se han mostrado rápidamente cambiantes en los últimos años, a tenor de los cambios drásticos acaecidos en el mercado de la vivienda. Por ejemplo, el aumento de los precios en la ciudad, y especialmente en Ciutat Vella, han estimulado una distribución de la inmigración más descentralizada en la que ganan protagonismo la periferia urbana y los municipios del área metropolitana. Igualmente, los problemas residenciales de los inmigrantes están hoy menos basados en los problemas de habitabilidad (infravivienda) que en otras manifestaciones de exclusión residencial, como los problemas de accesibilidad o las condiciones de ocupación de la vivienda (en especial, la sobreocupación).

En resumen, la investigación muestra como dos mecanismos opuestos pueden producir segregación residencial y diferencias residenciales. Muestra también como, en comparación con el caso de Barcelona, el sistema de vivienda en Amsterdam ha permitido unas condiciones residenciales para los grupos de origen inmigrante, por una parte, más acordes con sus preferencias y necesidades y, por otra, con una incidencia menor de la exclusión residencial.

3. El 23,5\% de hogares surinameses, el 37\% de los marroquíes y turcos, y el 16\% de los holandeses, reciben una media de 147,152 y 123 euros mensuales, respectivamente, en concepto de subvención al alquiler (encuesta sobre la demanda de vivienda WBO, 2003).

4. Pese a este escenario positivo, existen elementos de preocupación en los posibles efectos de las nuevas políticas de vivienda, así como en la situación de los inmigrantes ilegales. 


\section{Bibliografía}

Bordieu, P. (2000). Las estructuras sociales de la economía. Barcelona: Anagrama.

KeEsteloot, C.; Corie, C. (1998). «Housing Turks and Moroccans in Brussels and Amsterdam: Private and Public Markets». Urban Studies, 35, 10, 1835-1853. leal, J.; Allen, J.; Maloutas, T.; Padovani, L.; Barlow, J. (2004). Housing and welfare in Southern Europe. Londores: Blackwel.

Musterd, S.; Deurloo, R. (2002). "Unstable Immigrant Concentrations in Amsterdam: Spatial Segregation ans Integration of Newcomers». Housing Studies, $17,3,487-503$.

VAN AMERSFOORT, H.; CORTIE, C. (1996): «Social polarisation in a Welfare State? Immigrant in the Amsterdam Region». New Community, 4, 22, 671-687. 